Mass Profiles and Shapes of Cosmological Structures

G. Mamon, F. Combes, C. Deffayet, B. Fort (eds)

EAS Publications Series, Vol. ?, 2005

\title{
SOME SYSTEMATIC PROPERTIES OF ROTATION CURVES
}

\author{
McGaugh, S. ${ }^{1}$
}

\begin{abstract}
The rotation curves of spiral galaxies obey strong scaling relations. These include the Tully-Fisher and baryonic Tully-Fisher relations, and the mass discrepancy - acceleration relation. These relations can be used to place constraints on the mass-to-light ratios of stars. Once the stellar mass is constrained, the distribution of dark matter follows. The shape of the dark matter distribution is consistent with the expectations of NFW halos exterior to $1 \mathrm{kpc}$, but the amplitude is wrong. This is presumably related to the long-standing problem of the normalization of the Tully-Fisher relation and may imply a downturn in the amplitude of the power spectrum at small scales. More fundamentally, the persistent success of MOND remains a troubling fact.
\end{abstract}

\section{Introduction}

It is abundantly clear that there are mass discrepancies in the universe. Whether these imply the existence of invisible mass or a modification of dynamical laws is less clear. Either way, remarkable new physics must be involved.

The persistent flatness of rotation curves at radii well beyond the point where the observed baryon distribution predicts a declining $V(R)$ was one of the key observations that convinced us of the need for dark matter (e.g., Bosma 1981; Rubin et al. 1982). While the inference of dark matter is a perfectly natural one, strictly speaking the data merely indicate the presence of mass discrepancies. This can mean either dark matter or a breakdown in the equations relating mass and dynamics. Here I briefly review the application of rotation curves as a test of each of these cases in turn.

In principle, rotation curves provide an excellent probe of the radial force law in galaxies. Fig. 1 shows an example of an extended rotation curve. In practice, a number of issues complicate the use of rotation curves to constrain the mass distribution. These fall into two broad categories: issues concerning data quality

${ }^{1}$ Department of Astronomy, University of Maryland, College Park, MD, USA

(c) EDP Sciences 2021

DOI: (will be inserted later) 


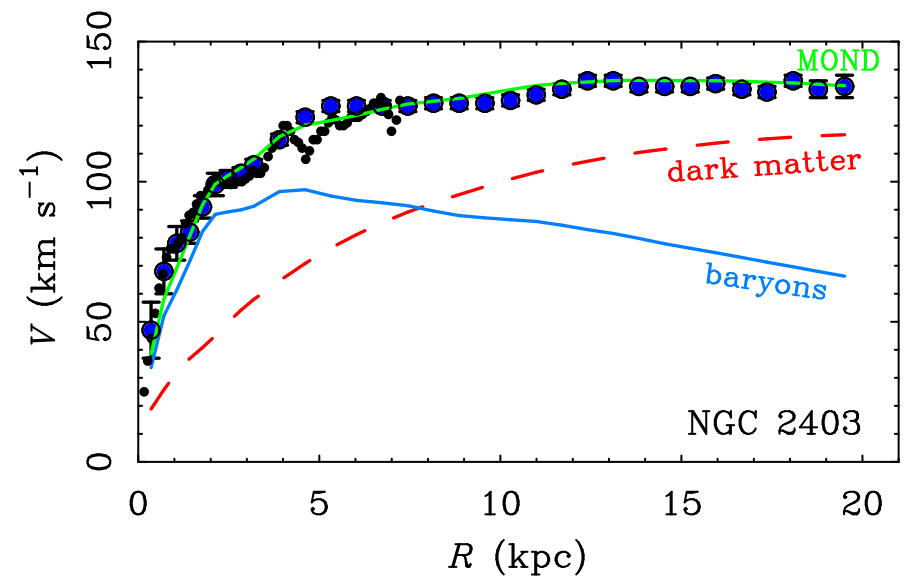

Fig. 1. Rotation curve and mass model of NGC 2403. The large points are $21 \mathrm{~cm}$ data from Begeman et al. (1991); the small points are H $\alpha$ Fabry-Perot data from BlaisOuellette et al. (2004). The declining solid line illustrates the contribution of the baryons (stars + gas); the dashed line that of the dark matter. These illustrate the combination of conventional components required to match the MOND fit (upper solid line).

(the accuracy with which the potential is traced, e.g., Swaters et al. 2003; de Blok et al. 2003) and the degeneracy of modeling dark and baryonic components. While there are always cases for which the data can be improved, the literature contains many excellent rotation curves that have been consistently measured by independent observers using different techniques. Yet even for perfect data, there is a terrible ambiguity between dark and luminous mass. The stellar mass is only known as well as the mass-to-light ratio $\Upsilon_{\star}$, and the inferred dark matter distribution can change dramatically for different $\Upsilon_{\star}$. This effect is much stronger than the uncertainties in the rotation curve itself.

\section{The Tully-Fisher Relation and Stellar Mass-to-Light Ratios}

For any individual galaxy, the degeneracy between dark and luminous mass is hard to lift. However, galaxies as a population obey a number of scaling relations (e.g., Persic \& Salucci 1988). We can test a variety of prescriptions for $\Upsilon_{\star}$; presumably the one that minimizes the scatter in relations like the baryonic Tully-Fisher relation is the one that comes closest to the truth.

McGaugh (2005) considered a variety of prescriptions: scaling $\Upsilon_{\star}$ as a fraction of maximum disk $\Upsilon_{\star}=\Gamma \Upsilon_{\max }$; relative to population synthesis models $\Upsilon_{\star}=$ $\mathcal{P} \Upsilon_{\text {pop }}$; and relative to the mass discrepancy - acceleration relation (the empirical version of MOND): $\Upsilon_{\star}=\mathcal{Q} \Upsilon_{a c c}$. These $\Upsilon_{\star}$ are shown as a function of color in Fig. [2

The prescription that is most consistent with stellar population synthesis mod- 

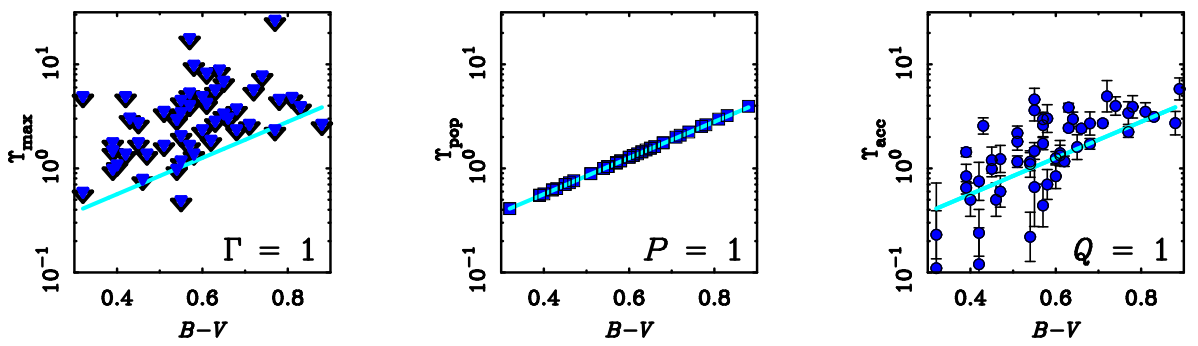

Fig. 2. Stellar mass-to-light ratios as a function of $B-V$ color. Left panel: maximum disk. Middle panel: stellar population synthesis (Bell et al. 2003). Right panel: $\Upsilon_{a c c}$ (MOND). The population model of Bell et al. (2003) is shown as a line in all three panels.

els is $\Upsilon_{a c c}$. Indeed, this is more consistent with such models than the models are with themselves, in that a realistic amount of scatter is built in. The case of $\mathcal{Q}=1$ also minimizes the scatter in the baryonic Tully-Fisher relation (Fig. 31) and, by construction, in the mass discrepancy - acceleration relation. The upshot of this is that the stellar mass-to-light ratio is well determined.
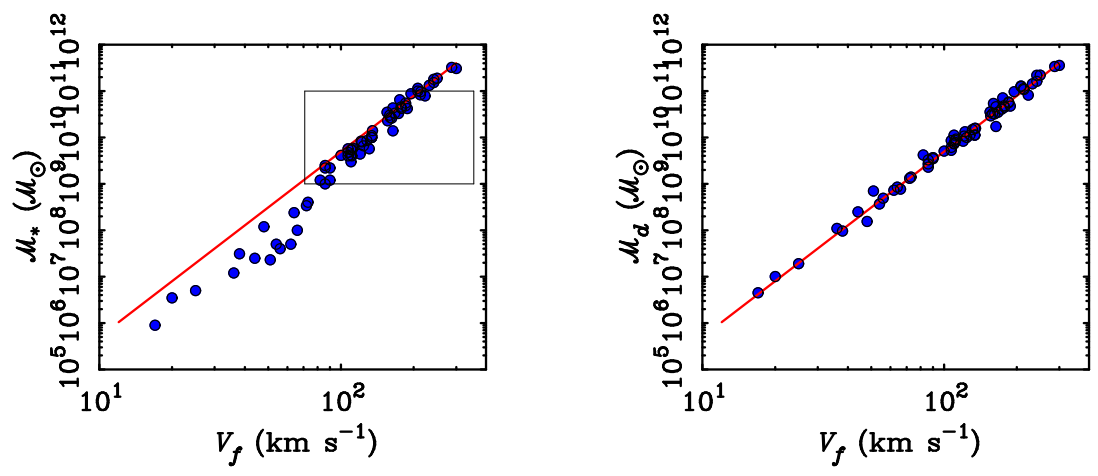

Fig. 3. Left panel: The stellar mass Tully-Fisher relation. Right panel: The baryonic Tully-Fisher relation (stars plus gas). The latter is well fit over five decades in mass by $\mathcal{M}_{d}=50 V_{f}^{4}$ (line). This is a considerably larger dynamic range than has been available until recently. The box in the left panel illustrates the range over which most Tully-Fisher work has been done.

\section{The Dark Matter Distribution}

Once $\Upsilon_{\star}$ is determined, the dark matter distribution follows. We need not limit consideration to the optimal case of $\mathcal{Q}=1$. For each prescription for $\Upsilon_{\star}$, we can ask what dark matter distribution is inferred. This is shown in Fig. [

When examined in detail, there are clear differences between the halo rotation 

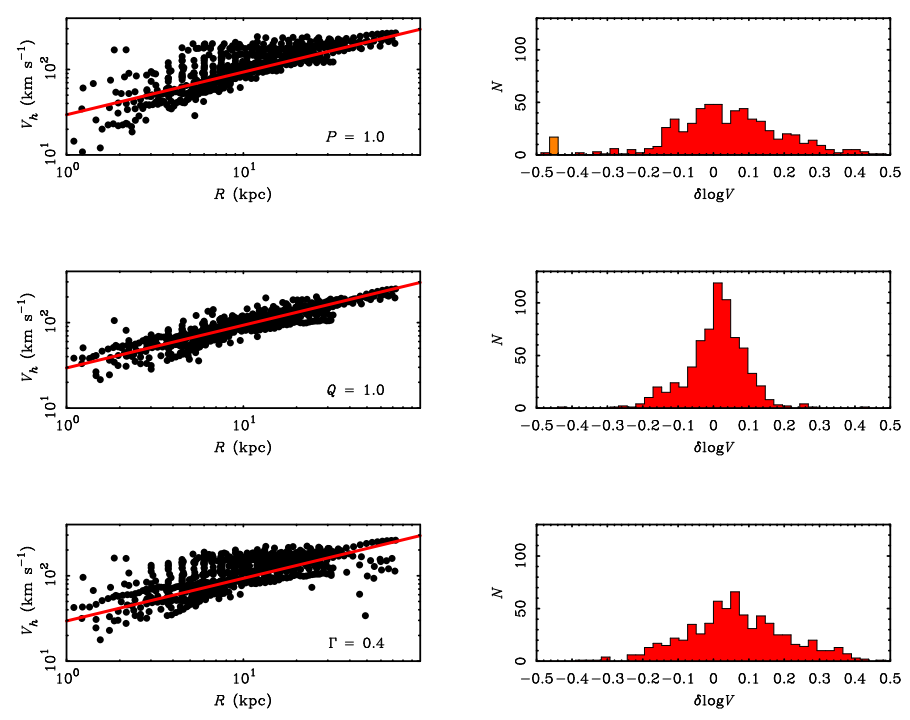

Fig. 4. Left panels: The inferred rotation curves of the dark matter halos (equivalent to the dashed line in Fig. 1) of sixty galaxies (with over 600 individual points) with high quality rotation curves (each point has a formal uncertainty of $5 \%$ or less). Each row illustrates a different prescription for the mass-to-light ratios of the stars. Right panels: the distribution of points around the solid line, which is the best fit to the case of minimum scatter (middle row).

curves of individual galaxies (Sancisi 2004; McGaugh 2004). However, when plot-

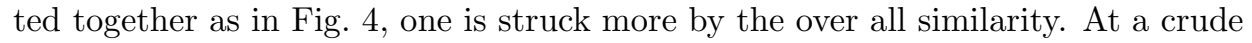
level, the data are consistent with all galaxies living in identical dark matter halos. Presumably, there is some mass spectrum of halos, but that is not obvious from these data.

Remarkably, the scatter in the inferred rotation curves is minimized for the case of $\mathcal{Q}=1$. The scatter in the dark matter component in this case is comparable to that expected in CDM simulations (Bullock et al. 2001). A fit to these data yield a line

$$
\log V_{h}=A+B \log R
$$

with $A=1.47$ and $B=0.49 \pm 0.01$. The value of the intercept $A$ depends on the choice of mass-to-light ratio, as tracked by the centroid of the distribution in the

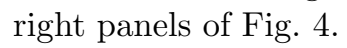

It is striking that the slope $B \approx 1 / 2$. This slope in velocity space corresponds to a density distribution $\rho \propto R^{-1}$, which is the inner slope of an NFW halo. This sheds no light on the cusp/core controversy, as the data here do not probe the inner kpc where that problem arises (de Blok 2004). It does, however, tell us about the density and structure of halos at intermediate radii.

What we observe is a continually rising halo rotation curve. We do not see the 

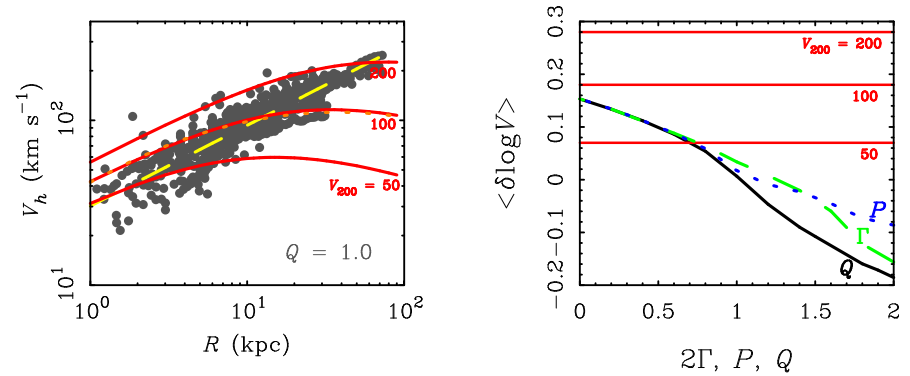

Fig. 5. Left panel: the inferred dark matter rotation curves for sixty galaxies tabulated in McGaugh (2005). The dashed line illustrates a slope of $1 / 2$, equivalent to a dark matter distribution whose density falls as $R^{-1}$. This is a good representation of the mean of the data for $R>1 \mathrm{kpc}$. The solid lines show NFW halos for several $V_{200}$ with concentrations as predicted by the vanilla $\Lambda$ CDM parameters of Tegmark et al. (2004). Also shown (dotted line) is the profile suggested by Navarro et al. (2004), which is virtually indistinguishable from NFW. Right panel: the variation of the mean velocity of the dark matter with different choices of mass estimator for the stars. The mean dark matter velocity increases as stellar mass declines, but hardly intersects with the region predicted by $\Lambda$ CDM (horizontal lines).

flat portion of an isothermal halo: the observed flat velocity is the combination of a declining baryonic component and a rising dark halo component. That $V_{f}$ remains roughly constant while its components vary with radius $\left[V_{f}^{2}=V_{b}^{2}(R)+V_{h}^{2}(R)\right]$ remains a curious puzzle.

The slope of the rising halo $V_{h}(R)$ is consistent with the expectation for an NFW halo. However, the amplitude is wrong, and the $B \approx 1 / 2$ slope persists rather too far. Over this range of radii, we expect to see significant curvature in the halo rotation curves (Fig. (5). Presumably, galaxies reside in halos of a variety of different mass halos, so perhaps we are merely seeing the locus of rising portions of the halo rotation curves. If this is the case, it is rather odd that we see so much of the rise but so little of the turn-over.

The observed amplitude of $V_{h}(R)$ poses a serious problem. CDM predicts velocities that are too high. This holds over the entire observed range of radii, irrespective of the detailed shape of the profile.

For vanilla $\Lambda \mathrm{CDM}$ parameters (Tegmark et al. 2004), we expect the dark matter velocity to be higher than observed by $\sim 0.25 \mathrm{dex}$. This is a large effect. In terms of cosmological parameters, the observations (for $\mathcal{Q}=1$ ) imply $\sigma_{8} \Gamma_{0.6} \approx 0.05$, where $\Gamma_{0.6} \propto \Omega_{m}^{0.6} h$ is a modified shape parameter (McGaugh, Barker, \& de Blok 2003). In contrast, vanilla $\Lambda \mathrm{CDM}$ has $\sigma_{8} \Gamma_{0.6}=0.24$.

Reconciling this difference would require large changes to basic cosmological parameters. This does not seem plausible. It may be sufficient to suppress the power spectrum on small scales, but it is quite a stretch to maintain consistency with other constraints. 
The most obvious solution is to reduce the stellar mass $(\mathcal{Q}<1)$. Fig. 5 shows how the centroid of the data varies with different scalings of $\Upsilon_{\star}$. The data just barely enter the region predicted by $\Lambda$ CDM in the limit $\Upsilon_{\star} \rightarrow 0$, and then only for rather lower mass halos than we typically associate with bright galaxies (which are well represented in this sample). We are not at liberty to decrease $\Upsilon_{\star}$ this much, as constraints on the IMF no longer permit arbitrarily small $\Upsilon_{\star}$. Moreover, the scatter in the baryonic Tully-Fisher and mass discrepancy - acceleration relations becomes unacceptably large for $\mathcal{P}<1 / 2$ (McGaugh 2004, 2005). Consequently, no matter how one chooses to look at the problem, and irrespective of the details of the slope of the halo (cusp or core), the velocity attributable to dark matter is significantly smaller than predicted by $\Lambda$ CDM.

\section{MOND and the Mass Discrepancy-Acceleration Relation}

In stark contrast to the situation for CDM, MOND fits rotation curves well (Fig. 6). That is does so is well established (Sanders \& McGaugh 2002). What this means is open to debate.

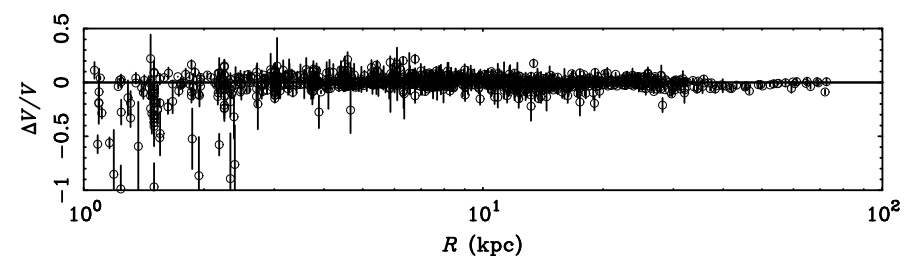

Fig. 6. Residuals of the MOND fits for 74 galaxies reviewed by Sanders \& McGaugh (2002). Data for all galaxies are plotted together. MOND fits to rotation curves are generally good. There is some tendency for MOND to over-fit the velocity at small radii, which can plausibly be attributed to non-circular motions and measurement difficulties (e.g., Swaters et al. 2003; de Blok et al. 2003). These are far smaller than what would be required to reconcile the data with the dense NFW halos predicted by $\Lambda$ CDM.

Like most astronomers, I ignored MOND for a long time, thinking it so unlikely as to not warrant consideration. I was obliged to reconsider when the fine-tuning problems with dark matter became severe (McGaugh \& de Blok 1998a), and the predictions of MOND came true in my data. Milgrom (1983) made a series of specific predictions for low surface brightness galaxies, all of which were subsequently realized (McGaugh \& de Blok 1998b).

This must mean something. The question, of course, is what. There is a widespread myth that MOND is somehow designed to fit rotation curves, and is guaranteed to do so. This is demonstrably false (de Blok \& McGaugh 1998). Quite the contrary, MOND fits have only one fit parameter, $\Upsilon_{\star}$, and are considerably better constrained than fits with dark halos. This is a non-trivial fact: if you write down the wrong force law, it fails (and fails badly) quite quickly. Many other modifications of gravity have been attempted, and suffered just such a fate. 

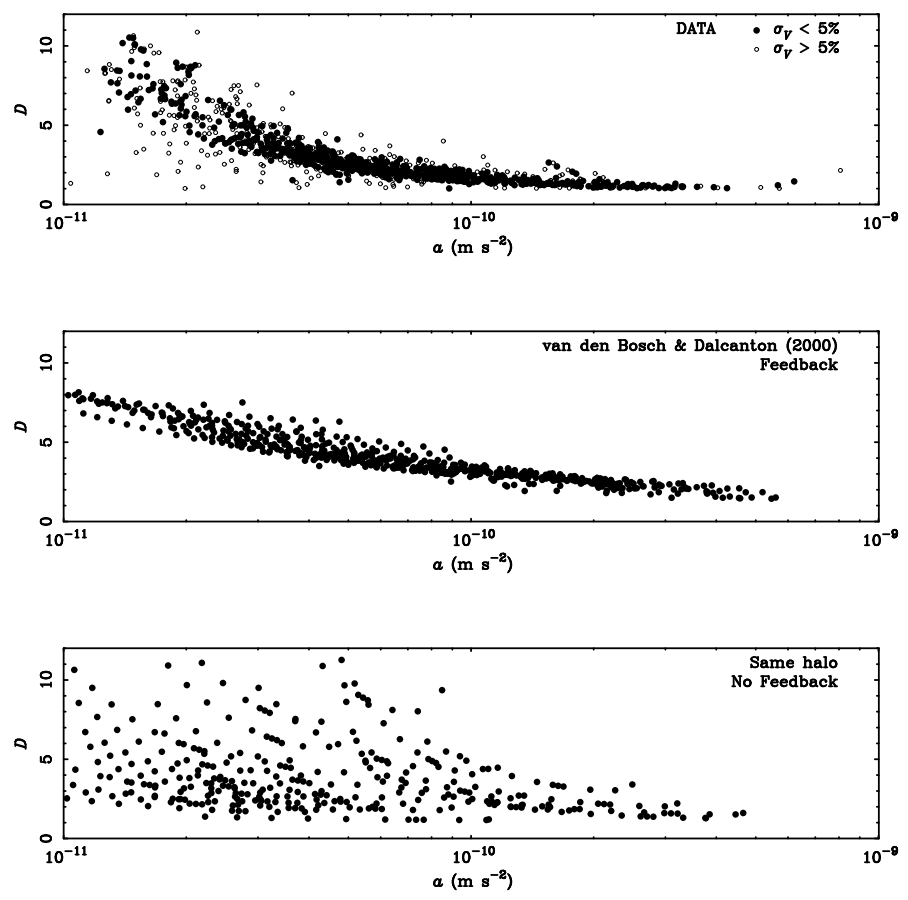

Fig. 7. The mass discrepancy $\mathcal{D}=V^{2} / V_{b}^{2}$ correlates with acceleration $a=V^{2} / R$. Top panel: Data: points with formal errors in velocity $<5 \%$ are shown as solid points, those with greater uncertainty as open points. The scatter is minimized (by construction) for MOND mass-to-light ratios, but the correlation persists for any plausible mass-tolight ratio (McGaugh 2004). Middle panel: The feedback model of van den Bosch \& Dalcanton (2000), which is to date the only attempt to quantitatively explain this aspect of the data with dark matter. Bottom panel: The 'same halo' model of McGaugh \& de Blok (1998a) illustrating the natural expectation for exponential disks residing in NFW halos sans feedback.

That MOND works as well as it does suggests that it is the correct effective force law, at least in spiral galaxies ${ }^{1}$.

We can rephrase MOND as a purely empirical relation (McGaugh 2005). The amplitude of the mass discrepancy is correlated with acceleration (Fig. 17). This mass discrepancy - acceleration relation is present in the data, whether we call it MOND or not. As such, it is an important empirical fact which we need to

\footnotetext{
${ }^{1}$ There is not space to review here the other successes of MOND, which are surprisingly numerous. That is not to say it is without problems. The most serious at present, to my mind, is the residual mass discrepancy in rich clusters of galaxies (Aguirre, Schaye, \& Quataert 2001; Sanders 2003). While it is tempting to use this to dismiss the whole idea, on balance it is not obvious that MOND is doing worse than CDM.
} 
understand.

I have devoted a good deal of time over the past decade trying to understand this relation in conventional terms. I have not succeeded. There are a few claims by others to have done so, the most serious being that of van den Bosch \& Dalcanton (2000). Their model is plotted together with the data in Fig. 7 as is a model I would consider natural ${ }^{2}$ for CDM. Many models are possible with dark matter, which provides no unique null hypothesis: there is a wide variety of things rotation curves might reasonably be expected to do. In MOND, there is precisely one thing rotation curves can do, and that is what they do. Philosophically, it is hard to imagine a stranger situation: why should dark matter look like MOND?

\section{References}

Aguirre, A., Schaye, J., Quataert, E. 2001, ApJ, 561, 550

Begeman, K.G., Broeils, A.H., \& Sanders, R.H., 1991, MNRAS, 249, 523

Bell, E.F., McIntosh, D.H., Katz, N., \& Weinberg, M.D. 2003, ApJS, 149, 289

Blais-Ouellette S., Amram P., Carignan C., \& Swaters R., 2004, A\&A, 420, 147

Bosma, A., 1981, AJ, 86, 1791

Bullock, J.S., Kolatt, T.S., Sigad, Y., Somerville, R.S., Kravtsov, A.V., Klypin, A.A., Primack, J.R., \& Dekel, A. 2001, MNRAS, 321, 559

de Blok, W.J.G. 2004, in IAU Symposium 220, ed. S.D. Ryder, D.J. Pisano, M.A. Walker, \& K.C. Freeman (San Francisco: Astronomical Society of the Pacific), 69

de Blok, W.J.G., Bosma, A., \& McGaugh, S.S. 2003, MNRAS, 340, 657

de Blok, W.J.G. \& McGaugh, S.S. 1998, ApJ, 508, 132

McGaugh, S.S., 2004, ApJ, 609, 652

McGaugh, S.S., 2005, ApJ, 632, 859

McGaugh, S.S., Barker, M.K., \& de Blok, W.J.G., 2003, ApJ, 584, 566

McGaugh, S.S., \& de Blok, W.J.G. 1998a, ApJ, 499, 41

McGaugh, S.S., \& de Blok, W.J.G. 1998b, ApJ, 499, 66

Milgrom, M. 1983, ApJ, 270, 371

Navarro, J.F., Frenk, C.S., \& White, S.D.M. 1997, ApJ, 490, 493

Navarro, J.F., et al., 2004, MNRAS, 349, 1039

Persic, M., \& Salucci, P. 1988, MNRAS, 234, 131

Rubin, V.C., Thonnard, N., \& Ford, W.K., 1982, ApJ, 238, 471

Sanders, R.H. 2003, MNRAS, 2003, 342, 901

Sancisi, R. 2004, in IAU Symposium 220, ed. S.D. Ryder, D.J. Pisano, M.A. Walker, \& K.C. Freeman (San Francisco: Astronomical Society of the Pacific), 233

Swaters, R.A., Madore, B.F., van den Bosch, F.C., \& Balcells, M. 2003, ApJ, 583, 732

Tegmark, M., et al., 2004, Phys. Rev. D, 69, 103501

van den Bosch, F.C., \& Dalcanton, J.J. 2000, ApJ, 534, 146

\footnotetext{
${ }^{2}$ Van den Bosch \& Dalcanton (2000) describe their model as natural. I do not think this word means what they think it means.
} 